\title{
Estudio del Rendimiento y Calidad de Frutos de Uvas de Mesa (Vitis vinifera $L$.) del Banco de Germoplasma del INPREX - Departamento de Tacna
}

\begin{abstract}
RESUMEN El presente trabajo de investigación tiene como objetivo principal determinar el rendimiento y calidad de frutos de siete variedades de vid, siendo estas, Red Globe, Italia Blanca, Alfonso Lavalle, Italia Negra, Gross Colman, Cardinal y Cereza. La variable principal de respuesta fue el peso de racimos por planta y por hectárea destacando en ello la variedad Red Globe, Alfonso Lavalle y Gross Colman con 43,329, 28,864 y 28,864 kg/ha respectivamente. Para la calidad del fruto, estuvo considerado el grado Brix y la concentración de la acidez total del mosto. El grado brix o sólidos solubles para la variedad Red Globe, Alfonso Lavallee y Gross Colman fue de $16.0,20.0$ y $15.0 \%$ respectivamente, como también los valores de la acidez total para las variedades referidas fue de 6.9, 6.7 y 7.2 gramos por litro de mosto. El diseño estadístico fue el de bloques completos al azar con 7 tratamientos y 3 repeticiones. El experimento se inició en el mes de agosto del 2008 y concluyó en febrero del 2009. Se investigaron en plantaciones de vid instaladas en sistemas de conducción en espaldera en cordón bilateral con distanciamientos de 2,0 m entre líneas y 1,5 m entre plantas, haciendo una densidad de 3,333 plantas por hectárea.
\end{abstract}

\begin{abstract}
This research paper, entitled "Study of Performance and fruit quality of table grapes (Vitis vinifera L.) Germplasm Bank of INPREX Department of Tacna. With the main objective of determining the yield and fruit quality of seven varieties of grapes, and these, Red Globe, Italia White, Alfonso Lavalle, Italy Black, Gross Colman, Cardinal and Cherry, the primary endpoint of response was the weight of clusters per plant and per hectare highlighting this as the Red Globe variety, Alfonso Lavalle and Gross Colman with 43.329, 28.864 and $28.864 \mathrm{~kg} / \mathrm{ha}$. respectively. For fruit quality, was considered the Brix concentration and total acidity of wine. The degree brix and soluble solids for the variety Red Globe, Alfonso Lavallee and Gross Colman was 16.0, 20.0 and $15.0 \%$ respectively, as well as the total acidity values for the varieties in question was 6.9, 6.7 and 7.2 grams per liter of must. The statistical design was a randomized complete block with 7 treatments and 3 replications. The experiment started in the month of August 2008 and concluded in February 2009. Were studied in vine plantings installed in piping systems in bilateral cordon trellis at a distance of $2.0 \mathrm{~m}$. between lines and $1.5 \mathrm{~m}$ between plants, with a density of 3.333 plants per hectare.
\end{abstract}

INTRODUCCIÓN. En nuestro pais la industria vitivinícola y la agroexportación de la uva de mesa va tomando importancia cada día más, debido a que el Perú va firmando el tratado de libre comercio con distintos paises del mundo.

Esto implica que las agroexportaciones tendrán que adecuarse a los estándares de calidad de los productos a exportar según paises compradores. La uva de mesa es un rubro muy importante para la agroexportacion peruana ya que nuestra costa peruana posee condiciones especiales para producir en forma satisfactoria el cultivo de la vid. En nuestro departamento contamos con un Banco de Germoplasma de vid, para uvas de mesa, piscos, vinos, pasas, etc., en el Instituto de Investigación Producción e Extensión Agraria (INPREX) de la Facultad de Ciencias Agrícolas de la Universidad Nacional Jorge Basadre Grohmann de Tacna. El propósito del presente trabajo experimental fue determinar el rendimiento y la calidad de uva de mesa en términos de grados brix o sólidos solubles y el porcentaje de acidez, ya que son los parámetros básicos para fijar la calidad de uva de mesa.

El departamento de Tacna puede potencializar su producción de vid con miras a la agroexportación a través de la uva de mesa y la industria vitivinícola.

\section{OBJETIVOS}

Objetivo General

Promover y potencializar el desarrollo de la viticultura en la zona.

Objetivo Específico

Determinar el rendimiento y calidad de frutos de uvas de mesa del Banco de Germoplasma del INPREX. 


\section{MATERIALES Y MÉTODOS}

\section{Ubicación del Campo Experimental}

El presente trabajo experimental se ejecutó en el Instituto de Investigación, Producción y Extensión Agraria (INPREX) de la Facultad de Ciencias Agrícolas de la Universidad Nacional Jorge Basadre Grohmann de Tacna, ubicado a $17^{\circ} 59^{\prime} 38^{\prime \prime}$ de latitud Sur, $70^{\circ} 14^{\prime} 24^{\prime \prime}$ de longitud Oeste a una altitud de 508 m.s.n.m.

La etapa de campo se dio inicio en el mes de agosto del 2008 con las podas y limpieza del campo experimental. La campaña agrícola duró hasta el mes de febrero del 2009 , concluyendo con las cosechas correspondientes y luego el procesamiento y los análisis de los datos obtenidos.

\section{Material Experimental}

El material vegetal objeto de estudio fueron siete variedades de uvas de mesa, instaladas en sistema de conducción en espaldera en cordón bilateral con distanciamientos de 2,0 m entre líneas, $1,5 \mathrm{~m}$. entre plantas, haciendo una densidad de 3,333 plantas/ha. Las variedades son las siguientes:

1. Red Globe

2. Italia Blanca

3. Alfonso Lavallee

4. Italia Negra

5. Gross Colman

6. Cardinal

7. Cereza

\section{Diseño Experimental}

El diseño experimental empleado fue el de bloques completos al azar (DBCA) con 7 tratamientos y 3 repeticiones.

\section{Variables de Respuesta}

$\mathrm{N}^{\circ}$ de racimos por planta

Peso de racimos por planta

Longitud de racimos

Diámetro de racimos

Calibre polar de bayas

Calibre ecuatorial de bayas

Grado Brix o sólidos solubles (\%)

Concentración de acidez total del mosto

\section{Análisis Estadístico}

El análisis de los datos de las variables de respuesta, se realizó utilizando la técnica del análisis de varianza, considerando el modelo del diseño estadístico de bloques y tratamientos aleatorizados y como estadístico de prueba se utilizó la prueba de " $F$ " a nivel de significación de 0,05 y 0,01 de probabilidad.

\section{RESULTADOS}

Cuadro 1: Análisis de varianza para número de racimos por planta.

\begin{tabular}{|c|c|c|c|c|c|}
\hline $\begin{array}{l}\text { Fuentes de } \\
\text { variación }\end{array}$ & $\begin{array}{c}\text { Grados de } \\
\text { libertad }\end{array}$ & $\begin{array}{l}\text { Suma de } \\
\text { cuadrados }\end{array}$ & $\begin{array}{c}\text { Cuadrado } \\
\text { medio }\end{array}$ & $\underset{\text { calculado }}{F}$ & $\begin{array}{l}F \text { tabular } \\
0,050,01\end{array}$ \\
\hline Bloques & 2 & $102,095^{\circ}$ & 51,047 & 3,331 & $3,88 \quad 6,93 \mathrm{~ns}$ \\
\hline Tratamientos & 6 & 807,238 & 134,539 & 8.779 & $3,00 \quad 4,82 \cdots$ \\
\hline Error & 12 & $1 ' 83,905$ & 15,325 & - & \\
\hline Total & 19 & 1093,238 & & & \\
\hline
\end{tabular}

CV: $23,692 \%$

significativo

ns: significativo ${ }^{* *}$ altamente

En el Cuadro 1, el análisis de varianza expresa que no existen diferencias estadísticas entre bloques, lo cual indica que los bloques fueron homogéneos.

Para el factor tratamiento se encontraron diferencias estadisticas significativas, lo cual nos indica que existe suficiente evidencia para creer que por lo menos uno de los tratamientos tuvo mayor efecto en el número de racimos por planta. El coeficiente de variabilidad fue de $23,692 \%$ que está indicando que la homogeneidad del material experimental utilizado es aceptable y que, por lo tanto, los datos experimentales son confiables.

Para determinar las verdaderas diferencias se realizó la prueba de significación de Duncan:

Cuadro 2: Prueba de significación de Duncan para el número de racimos por planta.

\begin{tabular}{|c|l|c|c|}
\hline O.M. & \multicolumn{1}{|c|}{ Tratamientos } & Promedio & $\begin{array}{c}\text { Significación } \\
\mathrm{a}=0,05\end{array}$ \\
\hline 1 & $\mathrm{~T}_{3}:$ Alfonso Lavalle & 26,33 & $\mathrm{a}$ \\
2 & $\mathrm{~T}_{5}:$ Gross Colman & 21,66 & $\mathrm{ab}$ \\
3 & $\mathrm{~T}_{4}:$ Italia negra & 21,33 & $\mathrm{ab}$ \\
4 & $\mathrm{~T}_{2}:$ Italia Blanca & 15,33 & $\mathrm{bc}$ \\
5 & $\mathrm{~T}_{1}:$ Red Globe & 12,33 & $\mathrm{c}$ \\
6 & $\mathrm{~T}_{6}:$ Cardinal & 10,00 & $\mathrm{c}$ \\
7 & $\mathrm{~T}_{7}:$ Cereza & 8,66 & $\mathrm{c}$ \\
\hline
\end{tabular}

Letras iguales no difieren estadisticamente $p<0,05$

El Cuadro 2, de la prueba de Duncan al 5\% señala que los tratamientos que alcanzaron el mayor promedio número de racimos fueron Alfonso Lavallee y Gross Colman con 26,33 y 21,66 racimos por planta respectivamente, los de menor promedio fueron Cardinal y Cereza con 10,00 y 8,66 racimos respectivamente. 
Cuadro 3: Análisis de varianza de peso de racimos por planta $(\mathrm{kg})$

\begin{tabular}{|c|c|c|c|c|c|}
\hline $\begin{array}{c}\text { Fuentes de } \\
\text { variacion }\end{array}$ & $\begin{array}{l}\text { Grados de } \\
\text { libertad }\end{array}$ & $\begin{array}{l}\text { Suma de } \\
\text { cuadrados }\end{array}$ & $\begin{array}{c}\text { Cuadrado } \\
\text { medio }\end{array}$ & $\begin{array}{c}F \\
\text { calculado }\end{array}$ & $\begin{array}{l}\text { F tabular } \\
0.05 \quad 0.01\end{array}$ \\
\hline Bloques & 2 & 9,201 & 4,600 & 2,283 & $3.886,93 \mathrm{~ns}$ \\
\hline Tratamientos & 6 & 161,371 & 26,895 & 13,444 & $3,00 \quad 4,82 \cdots$ \\
\hline Error & 12 & 24,185 & 2,015 & & \\
\hline Total & 19 & 194,757 & & & \\
\hline
\end{tabular}

CV: $19,160 \%$

**altamente significativo

En el Cuadro 3 el análisis de varianza expresa que no existen diferencias estadísticas entre bloques, lo cual indica que los bloques fueron homogéneos.

Para el factor tratamiento se encontraron diferencias estadísticas significativas, lo cual nos indica que existe suficiente evidencia para creer que por lo menos uno de los tratamientos tuvo mayor peso de racimos por planta. El coeficiente de variabilidad fue de $19,160 \%$ y está indicando que la homogeneidad del material experimental utilizado es aceptable y que, por lo tanto, los datos experimentales son confiables.

Para determinar las verdaderas diferencias se realizó la prueba de significación de Duncan.

Cuadro 4: Prueba de significación de Duncan para peso de racimos por planta $(\mathrm{kg})$.

\begin{tabular}{|c|l|c|c|}
\hline O.M. & \multicolumn{1}{|c|}{ Tratamientos } & Promedio & $\begin{array}{c}\text { Significación } \\
\alpha=0,05\end{array}$ \\
\hline 1 & $T_{1}:$ Red globe & 13,00 & $\mathrm{a}$ \\
2 & $\mathrm{~T}_{3}:$ Alfonsc Lavallee & 8,66 & $\mathrm{~b}$ \\
3 & $\mathrm{~T}_{5}:$ Gross Colman & 8,66 & $\mathrm{~b}$ \\
4 & $\mathrm{~T}_{6}$. Cardinal & 6,60 & $\mathrm{bc}$ \\
5 & $\mathrm{~T}_{2}:$ Italia blanca & 5,66 & $\mathrm{c}$ \\
6 & $\mathrm{~T}_{7}$. Cereza & 5,00 & $\mathrm{c}$ \\
7 & $\mathrm{~T}_{4}:$ Italia negra & 4,27 & $\mathrm{c}$ \\
\hline
\end{tabular}

Letras iguales no difieren estadisticamente $p<0,05$

El Cuadro 3, de la prueba de Duncan al 5\%, señala que los tratamientos que alcanzaron el mayor promedio fueron Red globe y Gross colman con 13,00 y $8,66 \mathrm{~kg}$ respetivamente y en el último se ubican la variedad cereza e Italia negra con 5,00 y $4,27 \mathrm{~kg} /$ planta respetivamente.

Cuadro 5: Análisis de varianza de longitud de racimos (cm)

\begin{tabular}{|l|c|c|}
\hline \multicolumn{1}{|c|}{ Tratamientos } & $\begin{array}{c}\text { Grado Brix o sólidos } \\
\text { solubles (\%) }\end{array}$ & $\begin{array}{c}\text { Acidez Total } \\
\text { grflitro de mosto }\end{array}$ \\
\hline 1. Red Globe & 16,0 & 6,9 \\
2. Italia Blanca & 18,4 & 7,0 \\
3. Alfonso Lavallee & 20,1 & 6,7 \\
4. Italia Negra & 22,8 & 6,5 \\
5. Gross Colman & 15,8 & 7,2 \\
6. Cardinal & 21,0 & 6,5 \\
7. Cereza & 17,5 & 6,8 \\
\hline
\end{tabular}

CV: $9,594 \%$

**altamente significativo
En el Cuadro 5, el análisis de varianza expresa que no existen diferencias estadísticas entre bloques, lo cual indica que los bloques fueron homogéneos.

Para el factor tratamiento se encontraron diferencias estadísticas significativas, lo cual nos indica que existe suficiente evidencia para creer que, por lo menos, uno de los tratamientos tuvo mayor longitud de racimos. El coeficiente de variabilidad fue de $9,594 \%$, está indicando que la homogeneidad del material experimental utilizado es aceptable y que, por lo tanto, los datos experimentales son confiables.

Para determinar las reales diferencias se realizó la prueba de significación de Duncan.

Cuadro 6: Prueba de significación de Duncan para longitud del racimo $(\mathrm{cm})$.

\begin{tabular}{|l|c|c|c|c|c|}
\hline $\begin{array}{c}\text { Fuentes de } \\
\text { variación }\end{array}$ & $\begin{array}{c}\text { Grados de } \\
\text { lbertad }\end{array}$ & $\begin{array}{c}\text { Suma de } \\
\text { cuadrados }\end{array}$ & $\begin{array}{c}\text { Cuadrado } \\
\text { medio }\end{array}$ & $\begin{array}{c}F \\
\text { calculado }\end{array}$ & $\begin{array}{c}\text { Ftabular } \\
0,050.01\end{array}$ \\
\hline Bloques & 2 & 2,00 & 1,00 & 0,160 & $3,886,93 \mathrm{~ns}$ \\
\hline Tratamientos & 6 & 477,33 & 79,55 & 12,785 & $3,004,82 *$ \\
\hline Error & 12 & 74,66 & 6,222 & & \\
\hline Total & 19 & 554,09 & & & \\
\hline
\end{tabular}

Fuente: Laboratorio de Química Analitica del INPREX 2009

El Cuadro 6, nos indica que la variedad de Red Globe destaca en el primer lugar con una longitud promedio de 34,00 , seguido de las variedades cereza, Alfonso Lavallee, Italia negra e Italia blanca que no presentan diferencias estadísticamente significativas en longitud de los racimos.

Cuadro 7: Análisis de varianza de diámetro de racimos (cm).

\begin{tabular}{|l|c|c|c|c|c|}
\hline $\begin{array}{l}\text { Fuentes de } \\
\text { varnación }\end{array}$ & $\begin{array}{c}\text { Grados de } \\
\text { libertad }\end{array}$ & $\begin{array}{c}\text { Suma de } \\
\text { cuadrados }\end{array}$ & $\begin{array}{c}\text { Cuadrado } \\
\text { medio }\end{array}$ & $\begin{array}{c}F \\
\text { calculado }\end{array}$ & $\begin{array}{c}\text { F tabular } \\
0,050.01\end{array}$ \\
\hline Bloques & 2 & 33,238 & 16,619 & 19,754 & $3,886,93 *$ \\
Tratamientos & 6 & 107,619 & 17,936 & 21,320 & $3,004,82 * *$ \\
Error & 12 & 10,095 & 0,841 & & \\
\hline Total & 19 & 150,952 & & & \\
\hline
\end{tabular}

En el Cuadro 7, el análisis de varianza indica diferencias altamente significativas entre bloques, tiene tratamientos, es decir, que el efecto de bloques tiene influencia en el diámetro de racimos, asimismo en cuanto a tratamientos existen diferencias en cuanto al diámetro de sus racimos. Para ver las reales diferencias se efectuó la prueba de significación de Duncan.

Cuadro 8: Prueba de significación de Duncan para diámetro deracimo $(\mathrm{cm})$.

\begin{tabular}{|l|l|c|c|}
\hline OM & Tratamientos & Promedio & $\begin{array}{c}\text { Significación } \\
\mathrm{A}=0.05\end{array}$ \\
\hline 1 & Red globe & 17,00 & $\mathrm{a}$ \\
2 & cereza & 16,00 & $\mathrm{a}$ \\
3 & Alfonsc Lavallee & 14,00 & $\mathrm{~b}$ \\
4 & Italia blanca & 13,33 & $\mathrm{~b}$ \\
5 & Italia negra & 11,33 & $\mathrm{c}$ \\
6 & Gross colman & 11,00 & $\mathrm{c}$ \\
7 & Cardinal & 11,00 & $\mathrm{C}$ \\
\hline
\end{tabular}

Letras iguales estadisticamente $p<0,05$ 
En el Cuadro 8, la prueba de significación de Duncan nos indica que la variedad Red Globe destaca en el primer lugar con un diámetro de su racimo de $17,00 \mathrm{~cm}$; seguido de la variedad cereza con $16,00 \mathrm{~cm}$; sin embargo, ambas variedades no presentan diferencias estadisticamente significativas. Asimismo, en el último lugar se ubican las variedades Gross Colman y Cardinal con diámetro de racimos de $11,00 \mathrm{~cm}$ respectivamente.

Cuadro 9: Análisis de varianza de calibre polar de bayas $(\mathrm{cm})$.

\begin{tabular}{|l|c|c|c|c|c|}
\hline $\begin{array}{l}\text { Fuentes de } \\
\text { variacion }\end{array}$ & $\begin{array}{c}\text { Grados de } \\
\text { libertad }\end{array}$ & $\begin{array}{c}\text { Suma de } \\
\text { cuadrados }\end{array}$ & $\begin{array}{c}\text { Cuadrado } \\
\text { medio }\end{array}$ & $\begin{array}{c}F \\
\text { calculado }\end{array}$ & $\begin{array}{c}\text { F labular } \\
0,050,01\end{array}$ \\
\hline $\begin{array}{l}\text { Bloques } \\
\text { Tratamientos }\end{array}$ & 6 & 0,298 & 0,139 & 2,701 & $3886,93 \mathrm{~ns}$ \\
Eror & 12 & 1,418 & 0,236 & 4,284 & 3,00482 \\
\hline Total & 19 & 2,377 & 5,516 & & \\
\hline CV: $9,485 \%$ & * significativo & & \\
\hline
\end{tabular}

Para el calibre polar de bayas o gramas de uva, el cuadro 9, de análisis de varianza, indica que no existen diferencias significativas entre entre tratamientos, es decir, que por lo menos una variedad difiere de las demás y para ver las reales diferencias se efectuó la prueba de significación de Duncan.

Cuadro 10: Prueba de significación de Duncan para calibre polar de bayas $(\mathrm{cm})$.

\begin{tabular}{|c|l|c|c|}
\hline M & Tratamientos & Promedio & $\begin{array}{c}\text { Significacion } \\
\mathrm{A}=0,05\end{array}$ \\
\hline 1 & Red globe & 3,06 & $\mathrm{a}$ \\
2 & Italia blanca & 2,47 & $\mathrm{~b}$ \\
3 & cereza & 2,47 & $\mathrm{~b}$ \\
4 & Alfonso Lavallee & 2,43 & $\mathrm{~b}$ \\
5 & Cardinal & 2,40 & $\mathrm{~b}$ \\
6 & Gross colman & 2,33 & $\mathrm{~b}$ \\
7 & italia negra & 2,16 & $\mathrm{~b}$ \\
\hline
\end{tabular}

En el Cuadro 10, la prueba de significación de Duncan nos indica que la variedad Red Globe destaca en el Primer lugar con un valor de $3,06 \mathrm{~cm}$ de diámetro de bayas: y la cual difiere significativamente de las demás variedad en estudio.

Cuadro 11: Análisis de varianza de calibre ecuatorial de bayas $(\mathrm{cm})$.

\begin{tabular}{|c|c|c|c|c|c|}
\hline $\begin{array}{l}\text { Fuentes de } \\
\text { variacion }\end{array}$ & $\begin{array}{c}\text { Grados de } \\
\text { libertad }\end{array}$ & $\begin{array}{l}\text { Suma de } \\
\text { cuaciados }\end{array}$ & $\begin{array}{l}\text { Cuadrado } \\
\text { medio }\end{array}$ & $\begin{array}{c}F \\
\text { calculado }\end{array}$ & $\begin{array}{l}\text { F tabular } \\
0,050.01\end{array}$ \\
\hline Blogues & 2 & 0,020 & 0,010 & 1,800 & $3,886,93 \mathrm{~ns}$ \\
\hline Tratamientos & 6 & 0,284 & 0,047 & 8,544 & $3,004,82^{* *}$ \\
\hline Error & 12 & 0,066 & 0,005 & & \\
\hline Total & 19 & 0,370 & & & \\
\hline
\end{tabular}

En el Cuadro 11, el análisis de varianza indica que no hace diferencias reales del efecto de bloques para la variable calibre ecuatorial de bayas o gramos de uva; pero existen diferencias altamente significativas entre los tratamientos, es decir, que por lo menos una variedad difiere de las demás en cuanto al calibre ecuatorial de bayas. Para ver las reales diferencias se efectuó la prueba de significación de Duncan.

Cuadro 12: Prueba de significación de Duncan para calibre ecuatorial de bayas $(\mathrm{cm})$.

\begin{tabular}{|c|l|c|c|}
\hline O.M & Tratamientos & Promedio & $\begin{array}{c}\text { Significación } \\
\mathrm{a}=0,05\end{array}$ \\
\hline 1 & Alfonso Lavallee & 3,06 & $\mathrm{a}$ \\
2 & Red Globe & 2,47 & $\mathrm{a}$ \\
3 & Gross Colman & 2,47 & $\mathrm{ab}$ \\
4 & Cereza & 2,43 & $\mathrm{ab}$ \\
5 & Cardinal & 2,40 & $\mathrm{~b} \mathrm{c}$ \\
6 & Italia blanca & 2,33 & $\mathrm{c}$ \\
7 & Italia negra & 2,16 & $\mathrm{c}$ \\
\hline
\end{tabular}

En el Cuadro 12 de la prueba de significación de Duncan para el calibre ecuatorial de las bayas o gramos de uva, se tiene que la variedad Alfonso Lavallee destaca en el primer del orden de mérito con $3,06 \mathrm{~cm}$, seguido de la variedad Red Globe; sin embargo, ambas variedad no presentan diferencias estadisticamente significativas. El último lugar lo ocupan las variables Italia blanca y la variedad Italia negra con valores de $2,33 \mathrm{~cm}$ y $2,16 \mathrm{~cm}$ respectivamente, y que ambas variedades no presentan diferencias significativas.

Cuadro 13: Grado Brix y contenido de acidez de la fruta.

\begin{tabular}{|l|c|c|}
\hline Tratamientos & Grado Brix (\%) & $\begin{array}{c}\text { Acidez Total } \\
\text { grfiltro de mosto }\end{array}$ \\
\hline 1. Red Globe & 16.0 & 6.9 \\
2. Italia blanca & 18.4 & 7.0 \\
3. Alfonso Lavallee & 20.1 & 6.7 \\
4. Italia negra & 22.8 & 6.5 \\
5. Gross Colman & 15.8 & 7.2 \\
6. Cardinal & 21.0 & 6.5 \\
7. Cereza & 17.5 & 6.8 \\
\hline
\end{tabular}

Fuente: Laboratorio de Quimica Analítica del INPREX 2009

Teniendo en cuenta que para las uvas de mesa el grado Brix mínimo es de $16 \%$, la variedad Gross Colman reporta $15,8 \%$, cifra por debajo de lo requerido como mínimo, y las variedades restantes reúnen las características deseables de la concentración de azúcar, destacando la variedad Italia negra con $22,8 \%$ de sólidos solubles o grados brix.

En cuanto a la acidez los términos varían para la uva de mesa de 6- 7 gramos por litro de mosto; en este caso solo la variedad Gross Colman ostenta mayor acidez y el resto reúne los requisitos para uva de mesa. 


\section{DISCUSIÓN}

En cuanto al rendimiento de fruto, podemos indicar que la variedad Red Globe se ubica en el primer lugar en el orden de mérito, si lo llevamos por hectárea tendríamos un valor de $43,329 \mathrm{~kg} / \mathrm{ha}$, ya que los distanciamientos son de 2,0 m. entre líneas, $1,5 \mathrm{~m}$ entre plantas y una densidad de 3,333 plantas/ha. Este valor está por encima del rendimiento nacional que varía $11,000-12,000 \mathrm{~kg} / \mathrm{ha}$.

La variedad Red Globe es una fruta de calidad muy aceptable por tener granos grandes de color rojo de buena conservación.

En el último lugar se ubica la variedad Italia negra con $4,27 \mathrm{~kg} /$ planta con $14,232 \mathrm{~kg} / \mathrm{ha}$, un rendimiento ligeramente superior al promedio nacional, pero con un grado de acidez mayor.

La cantidad y calidad están definidos por el rendimiento de la fruta y su concentración de azúcar (grado Brix) y contenido de acidez, por lo que nos permite determinar entre las mejores para uva de mesa al Red Globe, Alfonso Lavallee, Cardinal y la Italia blanca.

\section{CONCLUSIONES}

1.- Se concluye que la variedad Red Globe destaca en rendimiento, tamaño de granos y resistencia al almacenaje por poseer el hollejo grueso.

2.- En cuanto a la acidez, la concentración más baja la alcanzó la variedad Cardinal y la Italia negra.

3.- El contenido mayor de grado Brix lo reporta la variedad Italia negra, Cardinal y Alfonso Lavallee.

\section{REFERENCIAS BIBLIOGRÁFICAS}

Humberto I. MASÍAS HERNÁNDEZ. 1993. Manual, práctico de Viticultura.

INIPA -1982 Ministerio de Agricultura. El cultivo de la viden el Perú.

CAUVEL M y Reyner A. 1984. Manual de Viticultura. Ed. Mundo Prensa, Madrid.

CLEMENT J. et al. 1999. Cultivo de vid en espaldera Canarias.

CORDETACNA 1984. Curso Básico de Enología. Tacna, Perú.

CRESPY A. 1991. Viticultura de hoy. Ed. Hemisferio del Sur, Argentina.

IDALGO L. 1993. Tratado de Viticultura Genera.. Ed. Mundi-Prensa, Madrid.

MARIN F. 1989. Manual técnico de Viticultura. Tacna, Perú.

WINKLER A. 1985. viticultura. Ed. Continental S.A., México.

Minag 2007. Compendio estadístico aAgropecuario.

SÁNCHEZ NAVARRETE A, et al. 2007. Cooperación Empresarial para la Exportación de Uva de Mesa. Lima.

ROBLES, M. y ALFÉREZ, R. 2006. Investigación en Vid Italia Blanca UNJBG. Tacna, Perú. 
Variedades de Uva de Mesa

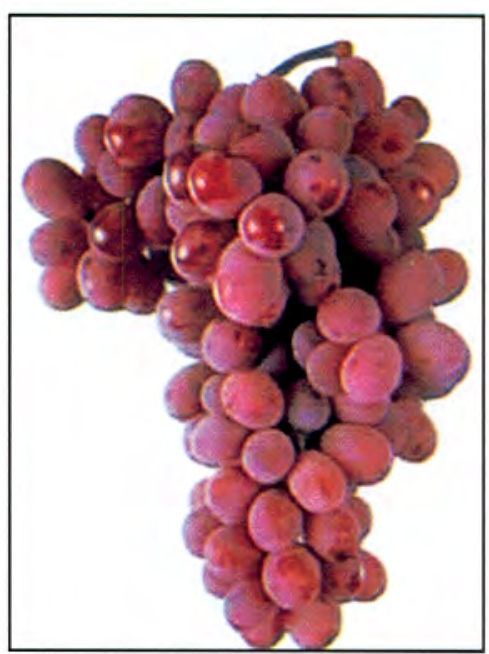

1. Red Globe

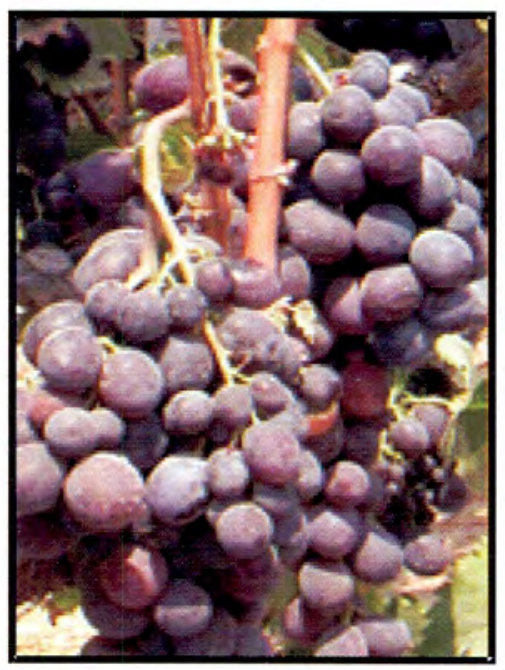

4. Italia Negra

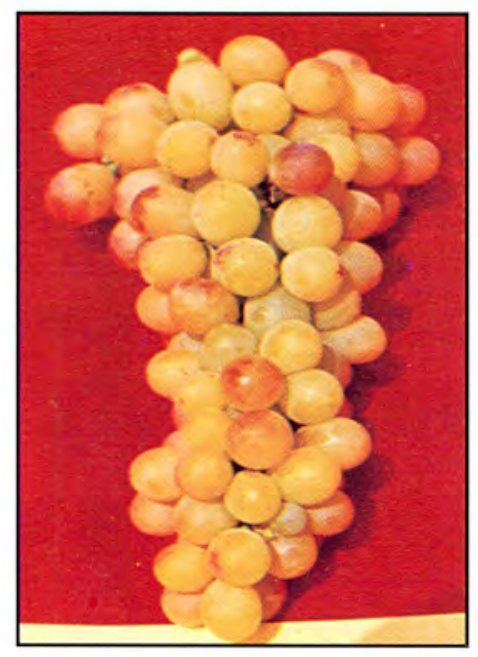

2. Italia Blanca

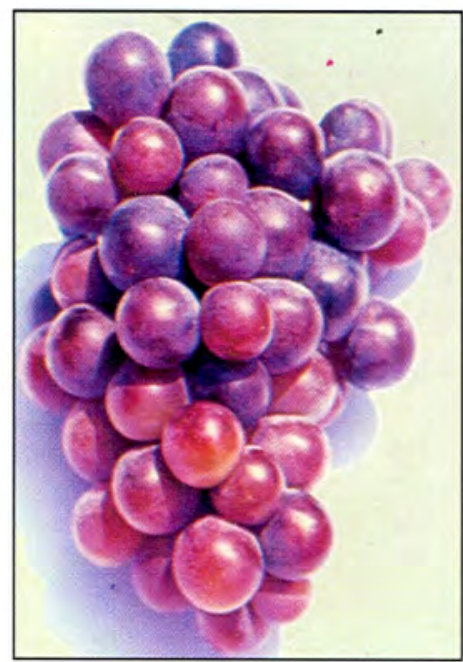

5. Gross Colman

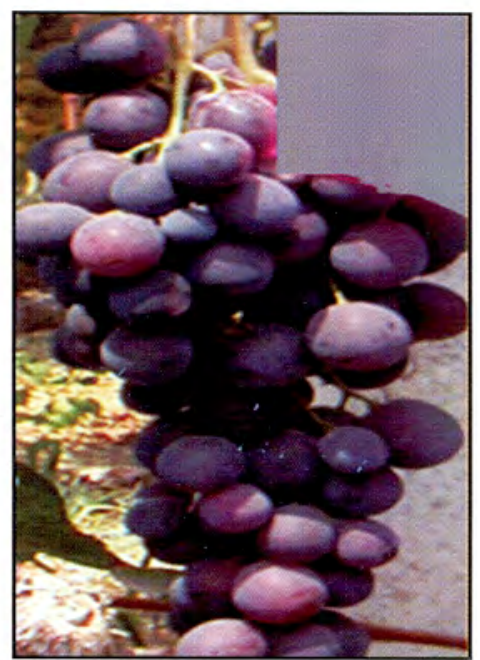

7. Cereza

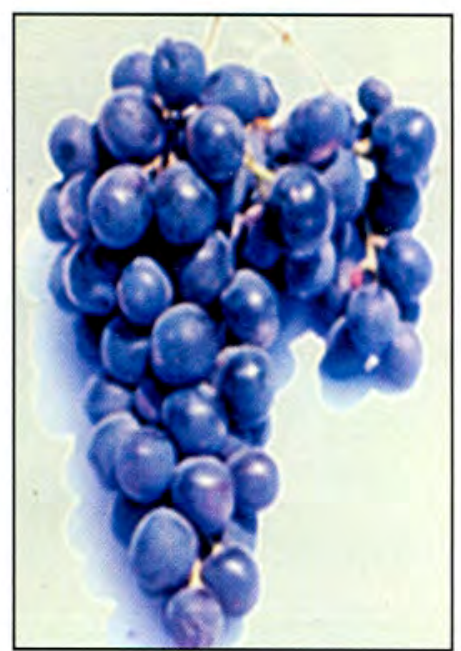

3. Alfonso Lavallee

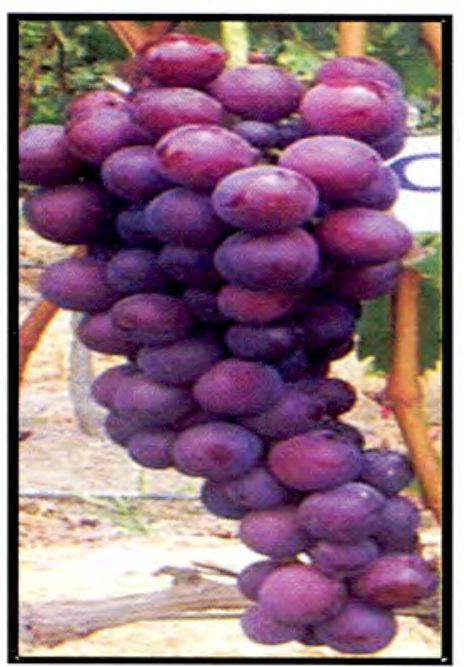

6. Cardinal 